\title{
Clinico-Mycological Study of Dermatophytoses at a Tertiary Care Hospital in Belagavi, Karnataka, India
}

\author{
Santhosh Gadadavar ${ }^{1 *}$, H.S. Shilpa ${ }^{2}$, C.S. Patil ${ }^{2}$, P.S. Vinay ${ }^{2}$ and Nirmala Shettar ${ }^{3}$ \\ ${ }^{1}$ Department of Microbiology JN Medical College Belagavi and Consultant Microbiologist, \\ KLE Centenary Hospital, Yallur Road, Belagavi, Karnataka, India \\ ${ }^{2}$ Department of Microbiology, USMKLE IMP Belagavi, Karnataka, India \\ ${ }^{3}$ Consultant Dermatologist, KLE Centenary Hospital, Yallur, Karnataka, India \\ *Corresponding author
}

\begin{abstract}
Keywords
Dermatophyte,

Tinea,

Trichophyton,

Microsporum

Article Info

Accepted:

16 April 2018

Available Online:

10 May 2018 species of Trichophyton, Microsporum and Epidermophyton. The present study was done to assess the clinical profile of dermatophytic infections and to identify the species of fungi that are prevalent in this region. A total of 80 samples consisting of skin scrapings, nail scrapings and hair follicles were collected from patients presenting with different ringworm/ tinea infections. Direct microscopy in $10 \%$ potassium hydroxide $(\mathrm{KOH})$ and culture on DTM and SDA was done for every sample. Tinea corporis 18/44 (40.9\%) was the most common presentation. Trichophyton spp were implicated in $97.7 \%(43 / 44)$ cases while Microsporum spp was detected only in $2.27 \%$ cases. Trichophyton mentagrophytes was the predominant organism (81.8\% cases) followed by Trichophyton rubrum $(11.36 \%$ cases). Tinea corporis was the most frequently encountered clinical condition followed by tinea cruris. T. mentagrophytes was implicated as the predominating species followed by T. rubrum and M. gypseum. Unhygienic conditions among low socio-economic group, occupation of construction work may be some of the contributing epidemiological factors.
\end{abstract}

\section{A B S T R A C T}

Dermatophytes are among the common fungal agents implicated in superficial skin infections worldwide. Infections commonly occur in hot and humid climates. They include

\section{Introduction}

Dermatophytes are fungi producing proteases that digest keratin and allow colonization, invasion and infection of the stratum corneum of the skin, hair shaft, and the nail (Gorbach et al., 2004). They are molds belonging to the three genera of fungi imperfecti: Microsporum, Trichophyton and Epidermophyton (Kanwar et al., 2001) Infection is generally cutaneous and restricted to the nonliving cornified layers because these fungi cannot penetrate the deeper tissues or organs of healthy immunocompetent host. (Bhatia and Sharma, 2014) The infection is commonly designated as ring worm or "tinea". The nomenclature of tenia infections is based on the location of the lesions on the body e.g. tinea capitis refers to ring worm infection of the head region. (Huda et al., 1995) Tinea infections are more prevalent in tropical and subtropical countries including India, where 
heat and moisture play an important role (Vernekar et al., 1991). In Asia, Trichophyton rubrum and Trichophyton mentagrophytes are most commonly isolated (Singh et al., 2016). Further, in India the cause of dermatophytoses is adversely influenced by economic factors like poverty, poor hygiene and social conditions like overcrowding. Nature of dermatophytoses may change with passage of time, living population, and evolution of preventive measures and hygienic conditions in society (Das et al., 2009; Venkatesan et al., 2007, Patwardhan and Dave, 1999).

Clinical lesions caused by these fungi are highly variable and closely resemble other skin diseases making laboratory diagnosis and confirmation necessary (Janardhan and Vani, 2017) Non-compliance to treatment, inadvertent use of steroids, irrational use of antifungals, sharing of clothes, geographic location predisposing to heat, humidity and increased sweating, obesity, associated conditions like diabetes, immunosuppression, atopy are all predisposing factors of chronic dermatophytosis. (Hay and Ashbee, 2016) Trichophyton rubrum is the commonest organism said to cause chronic dermatophytosis (Meriya Zacharia et al., 2017).

Frequent occurrence of fungal infections due to dermatophytes has been noticed in this region of Belagavi. Till now very few studies are done on dermatophytes in the state of Karnataka. The present study was done to assess the clinical and epidemiological profile of dermatophytic infections; to identify the species of fungi and to compare the clinical diagnosis with potassium hydroxide $(\mathrm{KOH})$ smear positivity and culture positivity.

It is necessary to make early laboratory diagnosis for better management of these conditions. The study was done utilizing conventional methods of isolation and identification of dermatophyte species from superficial mycoses in human patients.

\section{Materials and Methods}

The study population included 80 patients who were clinically suspected of dermatophytosis. The patients presented to the Dermatology outpatient department of KLE centenary charitable hospital, Yallur road, Belagavi. The study included samples collected from July 2017 to December 2017. A detailed clinical history including age, sex, socioeconomic status, occupation, duration of disease, history of recurrence and type of lesion, similar complaints in the family and contacts with animals or soil were elicited and recorded in all cases. General physical examination and systemic examination was conducted and investigations like hemoglobin, total count, differential count, blood sugar, and liver function test were done whenever necessary. Age limit and sex bias were not used and patients of all age groups and both the sexes were included. The exclusion criteria were: use of antifungal therapy (oral as well as topical) in the previous 3 months, immunocompromised state, secondarily infected, those who had taken other modality of treatment like steroids and presence of serious underlying systemic conditions as adjudged inappropriate by the clinician for inclusion in the study.

The patients were classified according to the site of involvement. These conditions included: Tinea corporis, T. capitis, T. cruris, $T$. pedis, $T$. unguium and $T$. manuum. For obtaining the samples aseptically, the infected areas or lesions were wiped with $70 \%$ ethanol in order to remove the dirt and environmental contaminants. Skin scraping, nail scrapings and clippings, hair stubs were collected from advancing margins of the lesions in sterile plastic containers with the help of sterile scalpel/ tweezers. $10 \% \mathrm{KOH}$ solution was 
used for skin and hair samples. 20\% KOH was used for nail samples. All preparations were examined under low power and confirmed under high power. The positive samples were processed for the isolation of the dermatophyte species on Sabouraud's Dextrose Agar (SDA, Himedia) and Dermatophyte test media (DTM, used as a selective media) containing cycloheximide $(0.05 \%)$ and chloramphenicol $(0.004 \%)$ under sterile conditions. The SDA and DTM slants were incubated at $30^{\circ} \mathrm{C}$ for four weeks and monitored for the growth. The colonies on the slants were examined for their morphology, texture and pigmentation (obverseand reverse). Slide culture was done to study the morphology in detail. Examination using Lactophenol Cotton Blue (LPCB) staining was done for every specimen which showed growth and observed under low (10× lens) as well as high power (40× lens) of light microscope. The identification was based on features such as organization of hyphae (pencil shaped, spiral, pyriform, septations etc.), microconidia and macroconidia (tear shaped, drop like, spherical, in bunches, abundance or rare etc.).

\section{Results and Discussion}

Out of 80 samples isolated $72(90 \%)$ were skin scraping, 07(8.75\%) were hairs stubs and 01 $(1.25 \%)$ was nail scraping. On cultural examination, 44(55\%) were found positive for dermatophyte species (Table 1 and Chart 1). Among different tinea conditions, tinea corporis $18 / 44(40.9 \%)$ figured at the top followed by tineacruris 14/44 (31.81\%) and tinea pedis $7 / 44(15.90 \%)$ for culture positivity (Figure 1 and 2).

Trichophyton species were implicated in 97.7\% (43/44) cases while Microsporum species was detected only in $2.27 \%$ cases. However, none of the Epidermophyton species was recovered in the present study. Among the
Trichophyton species, T. mentagrophytes was the predominant organism $(81.8 \%$ cases $)$ followed by $T$. rubrum ( $11.36 \%$ cases) (Table 2 and Figure 3). The identification of these dermatophyte species was based on cultural characteristics, growth rate, texture, colony size and pigmentation produced on obverse and reverse sides of SDA slants. $T$. mentagrophytes grew rapidly (3-5 days) on SDA, the growth was powdery to fluffy, cream to white on obverse and yellow to brown on reverse. On microscopic examination, well septate hyphae with numerous spherical microconida were visible. Spiral hyphae were seen (Figure $3 \mathrm{a}$ and $3 \mathrm{~b}$ ). $T$. rubrum grew relatively slower (10-15 days), the growth was powdery to velvety with reddish tinge on obverse and rusty brown to deep red on the reverse. Well septate, pencil shaped hyphae with numerous spherical microconidia along with macroconida were visible on microscopic examination (Figure 3c). M. gypseum grew rapidly (3-5 days), the growth was powdery to granular with rosy pink on obverse and yellow to brownish on reverse. Septate hyphae, rough thick walled macroconidia with rounded ends and 4-6 compartments were seen (Fig. 3d). $T$. mentagrophytes, the predominant species isolated was found associated mainly with Tinea corporis (15/36) $41.6 \%$ and Tinea cruris (12/36) $33.33 \%$. However, it was seen in all other tinea conditions also except $T$. manuum (Table 2). Among the culture positive cases, $79.54 \%$ were of males and $20.45 \%$ were of female patients. It was also observed that $70.45 \%$ patients fell in the age group of 20-50 years while $36.36 \%$ and $11.36 \%$ patients were in the age group of 1-20 years and > 50 years respectively (Table 3 ).

The present study highlights the clinical pattern and prevalence of different dermatophyte species implicated in different tinea infections in and around Belagavi city of Karnataka. 
Chart.1 Details of culture positive samples recovered from patients with different ring worm infections

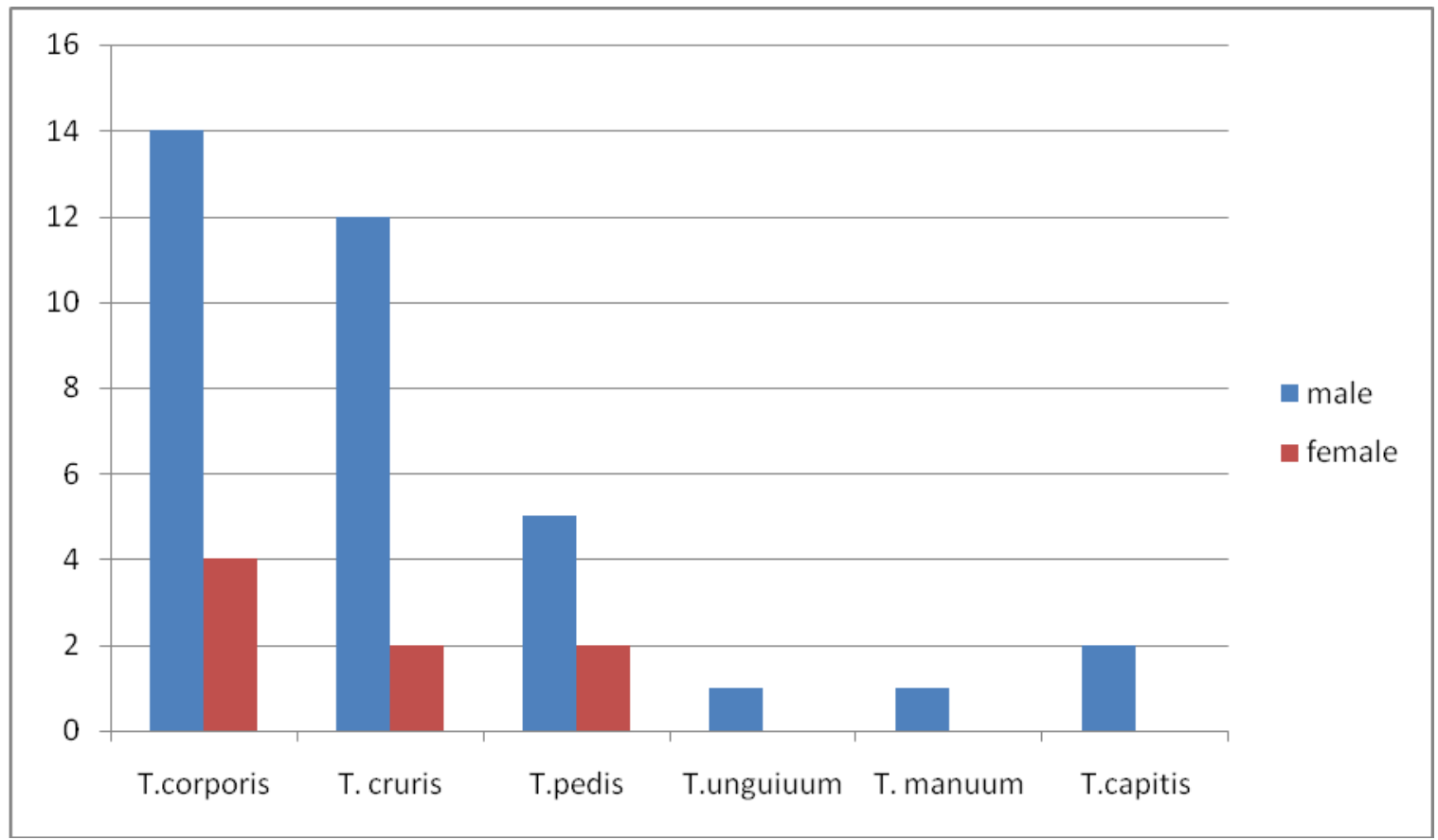

\begin{tabular}{|l|c|c|c|c|c|c|}
\hline Male & $\mathbf{1 4}$ & $\mathbf{1 2}$ & $\mathbf{5}$ & $\mathbf{1}$ & $\mathbf{1}$ & $\mathbf{2}$ \\
\hline Female & 4 & 2 & 2 & 0 & 0 & 1 \\
\hline
\end{tabular}

Table.1 Details of culture positive samples recovered from patients with different ring worm infections

\begin{tabular}{|l|c|c|c|c|c|c|c|}
\hline $\begin{array}{l}\text { Tinea infection } \\
\text { (types) }\end{array}$ & \multicolumn{3}{|c|}{ Samples examined (nos.) } & \multicolumn{2}{l|}{ KOH positive } & \multicolumn{2}{|c|}{ No of culture positive samples } \\
\hline T. corporis & Total & Male & Female & samples & Total & Male & Female \\
\hline T. cruris & 32 & 24 & 8 & 32 & 18 & 14 & 4 \\
\hline T. pedis & 24 & 20 & 4 & 24 & 14 & 12 & 2 \\
\hline T.unguium & 15 & 13 & 2 & 15 & 7 & 5 & 2 \\
\hline T. manuum & 1 & 1 & 0 & 1 & 1 & 1 & 0 \\
\hline T. capitis & 1 & 1 & 0 & 1 & 1 & 1 & 0 \\
\hline & 7 & 4 & 3 & 7 & 3 & 2 & 1 \\
\hline & 80 & 63 & 17 & 80 & 44 & $35(79.54 \%)$ & $9(20.45 \%)$ \\
\hline
\end{tabular}

Table.2 Association of dermatophyte spp. with different Tenia conditions

\begin{tabular}{|l|c|c|c|c|c|c|c|c|}
\hline Dermatophyte spp. & T.corporis & T.cruris & T.pedis & T.unguiuum & T.manuum & T.capitis & Total 44 & $\%$ \\
\hline T.mentagro-phytes & 15 & 12 & 6 & 1 & nil & 2 & 36 & 81.8 \\
\hline T.rubrum & 2 & 2 & 1 & nil & nil & nil & 5 & 11.36 \\
\hline T.tonsurans & nil & nil & nil & nil & nil & 1 & 1 & 2.27 \\
\hline T.verrucosum & nil & nil & nil & nil & 1 & nil & 1 & 2.27 \\
\hline M.gypseum & 1 & nil & nil & nil & nil & nil & 1 & 2.27 \\
\hline
\end{tabular}


Table.3 Details of sex and age group of patients of dermatophytosis examined

\begin{tabular}{|l|l|l|l|l|l|l|l|l|l|l|}
\multicolumn{3}{|c|}{ Male patients } & \multicolumn{3}{c|}{ Female patients } & \multicolumn{3}{c|}{ Distribution of culture positive patients } \\
into age groups
\end{tabular}

Fig.1 Clinical pictures of Tenia corporis

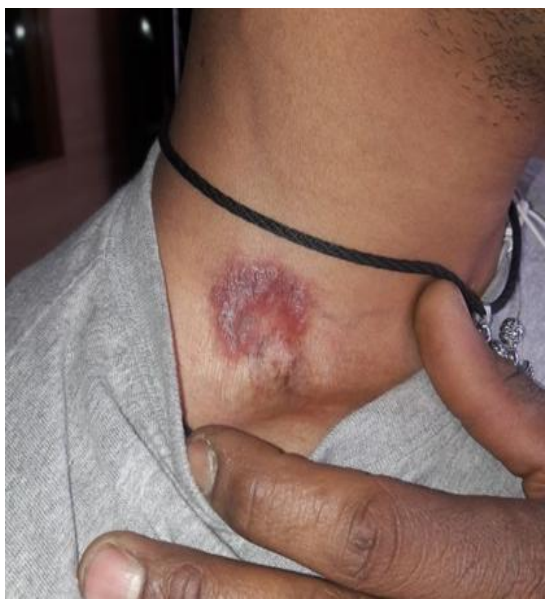

Figure 1a

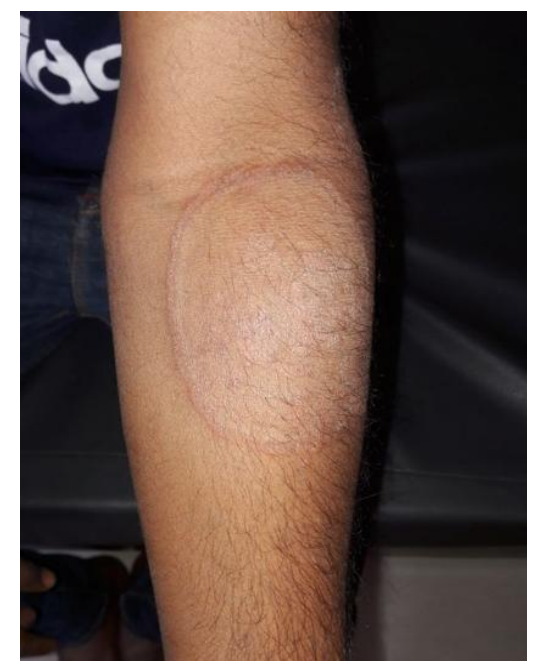

Figure 1c

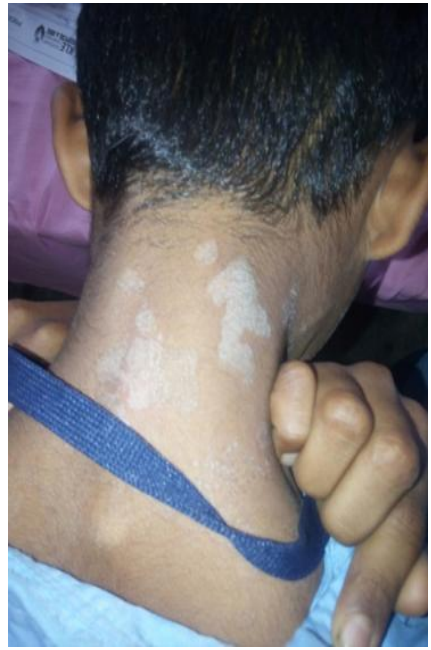

Figure $1 b$

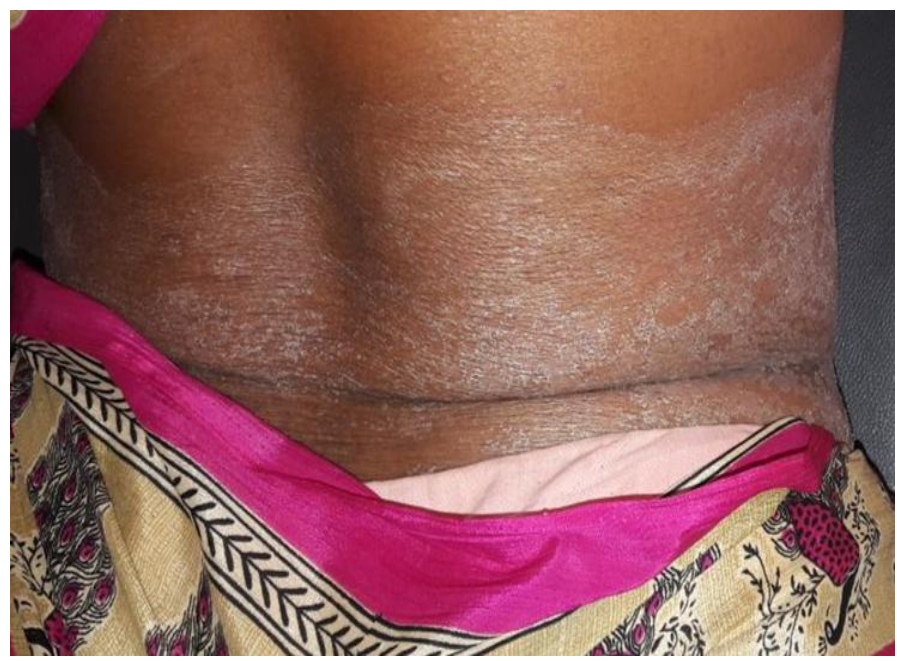

Figure 1d 
Fig.2 Clinical pictures of other teniaconditions

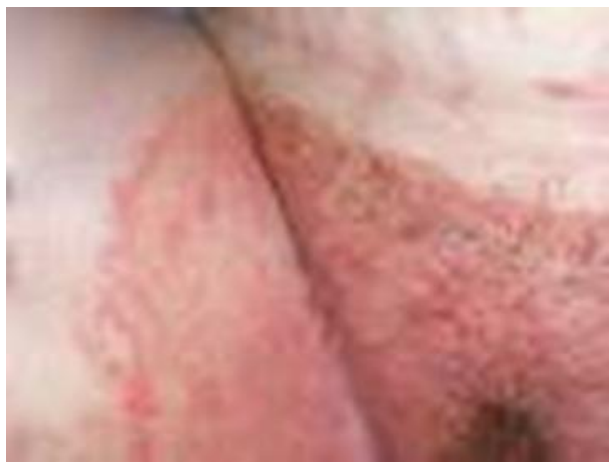

Figure 2a: Teniacruris

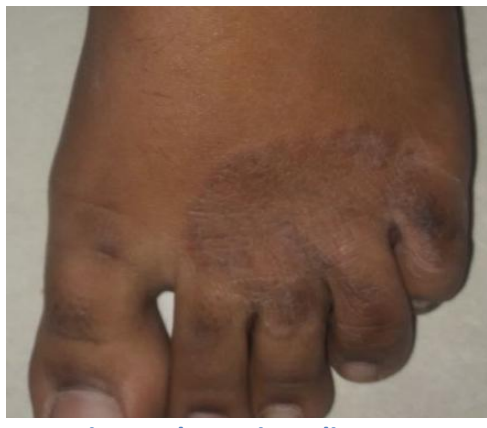

Figure 1b: Teniapedis

Fig.3 Microscopic pictures of the fungal isolates

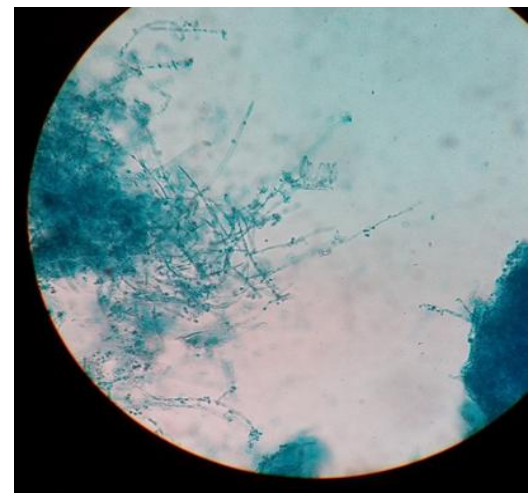

Figure 3a: T. mentagrophytes

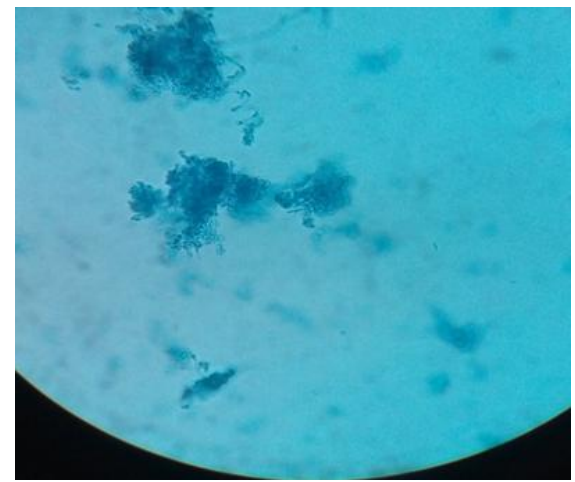

Figure 3b: Spiral hyphae of T. mentagrophytes

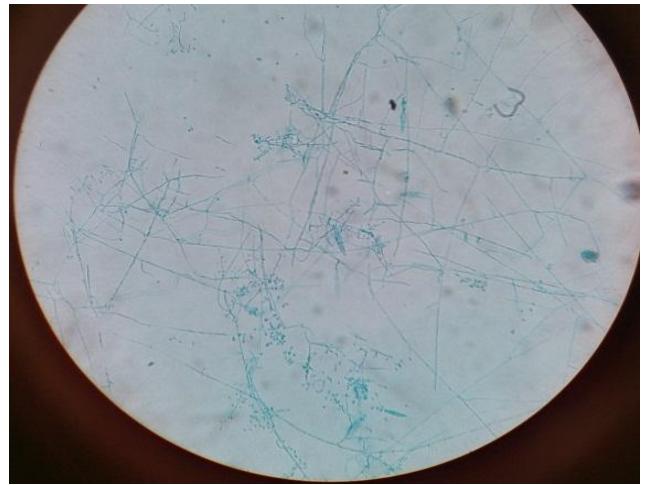

Figure 3c: T.rubrum: tear drop microconidia along hyphae, pencil shaped smooth walled macroconidia

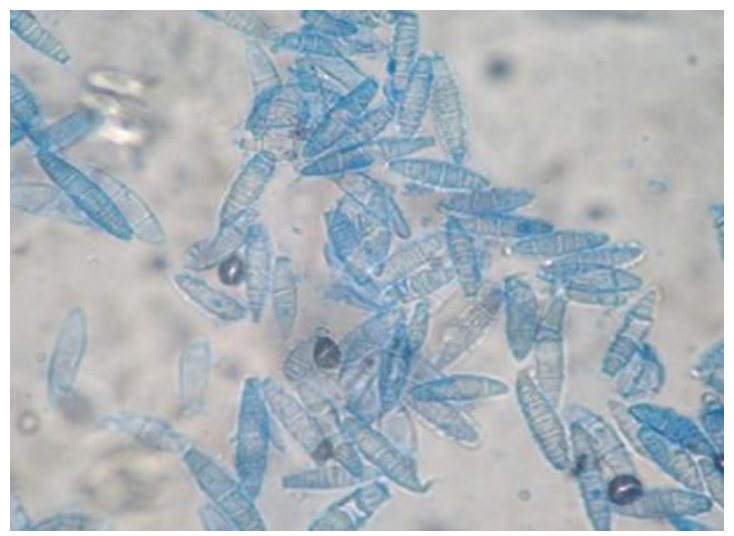

Figure 3d: M.gypseum - macroconidia with 4-6 compartments 
In general, hot and humid environment of the tropical and sub-tropical regions are best suited for the dermatophytic infections which have been reported from different parts of India. The climate in Belagavi (with latitude of $15.84^{\circ} \mathrm{N}$ and longitude of $74.49^{\circ}$ E) also remains hot and humid for major part of year which is favourable for growth of dermatophytic fungus. Hence the study was planned.

The study population consisted primarily of farmers and construction workers/laborers. Besides the climatic conditions favorable for the growth of dermatophytes, other factors such as overcrowding, unhygienic life style of the community with low socio-economic background were the contributing factors for the development of dermatophyte infections in this area.

Of the total 80 cases of superficial skin infections examined, only $44(55 \%)$ were culture positive. Although patients of all ages were susceptible to dermatophytosis, most (64.9\%) belonged to the age group of $21-50$ years as reported by other researchers (Bhatia and Sharma, 2014; Sarma and Borthakur, 2007; Patel et al., 2010, Agarwal et al., (2014); Hanumanthappa et al., 2012; Gupta et al., 2007). The probable reason for higher prevalence in this group could be that the individuals in this group are often most active because of their involvement in the outdoor activities.

The higher incidence may be due to increased physical activity and increased perspiration (Janardhan and Vani, 2017). Ofthe positive cases, $79.54 \%$ were males (Table 1). Such higher prevalence in males has been reportedin other parts of India as well as other countries of the world byseveral researchers (Bhatia and Sharma, 2014; Vernekar et al., 1991; Gupta et al., 2007; Poria and Samuel, 1981; Hanumanthappa et al., 2012; Sumathi et al., 2013; Agarwal et al., 2014; Bhavsar et al., 2012; Mathur et al., 2012; Jain et al., 2008). This may be due to increased outdoor physical activity and increased opportunity for exposure in men than women. Tinea corporis was the most common clinical condition observed in which various exposed parts of the body are affected followed by tinea cruris in which groin and surrounding areas are affected. The clinical picture of these conditions is presented through Figure 1. Similar observations have been made by other researchers (Venkatesan et al., 2007; Janardhan and Vani, 2017). Tinea conditions are consequence of exhaustive physical work and prolonged exposure to sun leading to excessive sweating. Among the females who had tinea corporis, common site involved was the waist area due to the patterns of clothing worn by the women like the saree and salwar suits which act as precipitating factors due to friction, increased sweating, collection of dust particles and fungal spores at belt line.

In addition, the tight fittings and synthetic clothing particularly in males provide damp, sweaty and warm skin conditions. All these factors favour the growth of dermatophytes (Ranganathan et al., 1995; Singh and Beena 2003). Tinea pedis and tinea unguium might result from wearing of socks and shoes for a long period providing damp conditions especially in inter-digital spaces.

In the present study, T. mentagrophytes was the predominant dermatophyte $(81.8 \%)$ involved followed by $T$. rubrum (11.3\%). This finding is contrary to the observations of others in which a reverse trend has been reported (Balakumar et al., 2012; Patel et al., 2010; Pandey and Pandey 2013). This could be due to the fact that $T$. rubrum is generally linked to chronic dermatophytosis (Aya et al., 2004). However, we do not have exact data about the chronic cases of dermatophytosis as they were excluded on the basis of history extracted from the patients. Therefore, the low proportion of $T$. rubrum might be involved in acute superficial mycosis. Also, as this organism is a slow growing organism, there is a possibility that other dermatophyte species might overgrow or mask the growth of T. rubrum while attempting 
isolation. PCR amplification directly from the samples could be a better tool to prove this. Molecular methods were not used for the diagnosis in our study due to the limitation of resources. Microsporum gypseum was involved only in $2.27 \%$ cases. We, however, did not observe any involvement of Epidermophyton spp in the present study. It may be concluded from the present study that the climatic conditions of Belagavi city favour infections caused by dermatophyte fungi. Dermatophytosis is mainly a disease of young and middle age adults, particularly males due to chances of exposure to the risk factors are more due to physical exertion and contact with infected persons. Patients from lower socioeconomic group predominated the study group due to poor personal hygiene, overcrowding and poor nutritional status. Tinea corporis and Tinea cruris were the most common clinical types. Although, the present study is a random study that focuses primarily on the prevalence of different dermatophyte species in a small area in the Belagavi city, more systematic study covering larger population and over a longer period of time would give a better insight into the epidemiology of dermatophytosis in the area.

\section{Consent}

Written informed consent was obtained from the patients for the publication of this report and any accompanying images.

\section{Competing Interests}

The authors declare that they do not have any competing/conflicting interests.

\section{Acknowledgements}

Authors express their sincere thanks to Dr Sheetal Harakuni, Professor of Microbiology for helping in the identification of species and Mr. Stephen Amathi, Laboratory technician for assisting in collection and processing of samples.

\section{References}

Agarwal US, Saran J, Agarwal P. 2014. Clinico mycological study of dermatophytoses in a Tertiary care centre in North West India at S.M.S. Medical College, Jaipur, Rajasthan.Indian Journal of Dermatology Venereology and Leprology 80(2), 194.

Aya S, Jose RFM, Maria EHM, Mattilde R, Nancy AG, Celso JG, Mauricio MO, Lphis C, Clarisse Z. 2004. HLA in Brazilian Ashkenazic Jews with chronic dermatophytosis caused by Trichophyton rubrum. Brazilian Journal of Microbiology 35, 69-73.

Balakumar S, Rajan S, Thirunalasundari T, Jeeva S. 2012. Epidemiology of dermatophytosis in and around Tiruchirapalli, Tamilnadu, India. Asian Pacific Journal of Tropical Diseases 2(4), 286-289.

Bhatia VK and Sharma PC. 2014. Epidemiological studies on Dermatophytosis in human patients in Himachal Pradesh, India. Springer Plus 3,134 .

Bhavsar HK, Modi DJ, Sood NK, Shah HS. 2012. A study of superficial mycoses with clinical mycological profile in tertiary care hospital in Ahmedabad, Gujarat. National Journal of Medical Research 2(2):160-164.

Das K, Basak S, Ray S. 2009. A study on superficial fungal infection from West Bengal: A brief report. Journal of Life Sciences 1(1), 51-55.

Gorbach SL, Bartlett JL, Blacklow NR.2004. Infectious Disease. In: Lippincott Williams and Wilkins; 3rd ed. Philadelphia, 1162-80.

Gupta M, Sharma NL, Kanga AK, Mahajan VK, Tegta GR.2007. Onychomycoses: Clinicomycologic study of 130 patients from Himachal Pradesh, India. Indian Journal of Dermatology Venereology and Leprology 73(6), 389-392.

Hanumanthappa H, Sarojini K, Shilpasree P, Muddapur SB. 2012. Clinico mycological study of 150 cases of Dermatophytosis in a Tertiary Care Hospital in South India. Indian Journal of Dermatology 57(4), 3223.

Hay RJ, Ashbee HR. 2016. Fungal infections. In: Griffiths CEM, Barker J, Bleiker T, et al., 
eds. Rook's textbook of dermatology. 9th edn. Oxford: Wiley-Blackwell, 32.1-32.55.

Huda MM, Chakraborthy N, Bordoloi JNS. 1995. A clinico-mycological study of superficial mycoses in upper Assam. Indian Journal of Dermatology Venereology and Leprology61, 329-332.

Jain N, Sharma M, Saxena VN.2008.Clinicomycological profile of dermatophytosis in Jaipur, Rajasthan. Indian Journal of Dermatology Venereology Leprology 74(3), 274-5.

Janardhan B, Vani G. 2017. Clinico mycological study of dermatophytosis. International Journal of Research in Medical Sciences 5, 31-39.

Kanwar AJ, Mamta, Chander J. 2001. Superficial fungal infections. In: Valia GR, ed. IADVL Text bookand Atlas of Dermatology. 2nd ed. Mumbai: Bhalani Publishing House, 215-258.

Mathur M, Kedia SK, Ghimire RBK. 2012. Epizoonosis of Dermatophytosis: A clinico mycological study of Dermatophytic infections in central Nepal. Kathmandu University Medical Journal 37(1), 30-33.

Pandey A, Pandey M. 2013. Isolation and characterization of dermatophytes with tinea infection at Gwalior, Madhya Pradesh, India. International Journal Pharmaceutical Investigation 2(2), 05-08.

Patel P, Mulla S, Patel D, Shrimali G.2010.A study of superficial mycosis in south Gujarat region. National Journal of Community Medicine 1(2), 85-88.

Patwardhan N, Dave R.1999. Dermatomycosis in and around Aurangabad. Indian Journal of Pathology and Microbiology 42, 455-462.

Poria VC, Samuel A. 1981. Dermatomycoses in and around Jamnagar. Indian Journal of Dermatology Venereology Leprology 47(2), 84-87.
Ranganathan S, Menon T, Selvi GS, Kamalam A. 1995. Effect of socio-economic status on the prevalence of dermatophytosis in Madras. Indian Journal of Dermatology Venereology Leprology 61, 16-18.

Sarma S, Borthakur AK. 2007. A Clinico Epidermatological study of dermatophytoses in Northeast India. Indian Journal of Dermatology Venereology Leprology 73(6), 427-8

Singh S, Beena PM. 2013. Profile of dermatophyte infections in Baroda. Indian Journal of Dermatology Venereology Leprology 69, 281-283.

Singh S, Kumar A, Agrawal A. 2016. Study of dermatophytes and incidence of different clinical types of tinea in skin OPD. Eastern Journal Medical Sciences 1(1), 24-30

Sumathi S, Mariraj J, Shafiyabi S, Ramesh R, Krishna S. 2013. Clinico mycological study of dermatophytoses at Vijayanagar Institute of Medical Sciences (VIMS), Bellary. International Journal of Pharmaceutical and Biomedical Research 4(2), 132-134.

Venkatesan G, Singh AJA, Murugesan AG, Janaki C, Shankar SG. 2007. Trichophyton rubrum - the predominant aetiological agent in human dermatophytosis in Chennai, India. African Journal of Microbiology Research 1(1), 9-12.

Verenkar MP, Pinto MJ, Rodrigues S, Roque WP, Singh I. 1991. Clinico-microbiological study of dermatophytoses. Indian Journal of Pathology and Microbiology 34(3), 186192.

Zacharia M, Kunjukunju BP.2017. Clinical profile of patients with chronic dermatophytosis-A descriptive study from a tertiary care centre in Kerala. Journal of Evidence Based Medicine in Healthcare 4(47), 2863-2866.

\section{How to cite this article:}

Santhosh Gadadavar, H.S. Shilpa, C.S. Patil, P.S. Vinay and Nirmala Shettar. 2018. ClinicoMycological Study of Dermatophytoses at a Tertiary Care Hospital in Belagavi, Karnataka, India. Int.J.Curr.Microbiol.App.Sci. 7(05): 1872-1880. doi: https://doi.org/10.20546/ijcmas.2018.705.220 